\title{
Evolution equations in Fréchet spaces
}

\author{
Saïd Abbas ${ }^{\mathrm{a}}$, Amaria Arara ${ }^{\mathrm{b}}$, Mouffak Benchohra ${ }^{\mathrm{c} *}$ and Fatima Mesri ${ }^{\mathrm{d}}$ \\ ${ }^{a}$ Laboratory of Mathematics, Geometry, Analysis, Control and Applications, Tahar Moulay University of Saïda, P.O. Box 138, EN-Nasr, \\ 20000 Saïda, Algeria \\ ${ }^{\mathrm{b}}$ Laboratory of Mathematics, Djillali Liabes University of Sidi Bel-Abbès, P.O. Box 89, Sidi Bel-Abbès 22000, Algeria \\ ${ }^{\mathrm{c}}$ Laboratory of Mathematics, Djillali Liabes University of Sidi Bel-Abbès, P.O. Box 89, Sidi Bel-Abbès 22000, Algeria \\ ${ }^{\mathrm{d}}$ Laboratory of Mathematics, Djillali Liabes University of Sidi Bel-Abbès, P.O. Box 89, Sidi Bel-Abbès 22000, Algeria \\ *Corresponding author E-mail: benchohra@univ-sba.dz
}

\section{Article Info}

Keywords: Densely defined operator, evolution system, fixed point, evolution equation, mild solution.

2010 AMS: $34 G 20,34 G 25$.

Received: 30 April 2018

Accepted: 14 May 2018

Available online: 27 May 2018

\begin{abstract}
This paper deals with the existence of mild solutions for a class of evolution equations. The technique used is a generalization of the classical Darbo fixed point theorem for Fréchet spaces associated with the concept of measure of noncompactness.
\end{abstract}

\section{Introduction}

There has been a significant development in functional evolution equations in recent years; see the monographs $[2,3,14,17,18]$ and the references therein. By means of a nonlinear alternative of Leray-Schauder type for contraction operators on Fréchet spaces [13], Baghli and Benchohra [4, 5] provided sufficient conditions for the existence of mild solutions of some classes of evolution equations, while in [6, 7, 8] the authors presented some global existence and stability results for functional evolution equations and inclusions in the space of continuous and bounded functions. In [1], an iterative method is used for the existence of mild solutions of evolution equations and inclusions. Using the Tichonov's fixed point theorem, Olszowy and Wȩdrychowicz [16] considered a class of evolution equations on unbounded intervals. However in the previous papers some restrictions like, the compactness of the semigroup, the Lipschitz condition on the nonlinear term or the boundedness of the obtained mild solutions, are supposed.

In the present paper, we discuss the existence of mild solutions for the evolution equation

$$
u^{\prime}(t)=A(t) u(t)+f(t, u(t)) ; \text { if } t \in \mathbb{R}_{+}:=[0, \infty),
$$

with the initial condition

$$
u(0)=u_{0} \in E
$$

where $f: \mathbb{R}_{+} \times E \rightarrow E$ is a given function, $(E,\|\cdot\|)$ is a (real or complex) Banach space, and $\{A(t)\}_{t>0}$ is a family of linear closed (not necessarily bounded) operators from $E$ into $E$ that generate an evolution system of bounded linear operators $\{U(t, s)\}_{(t, s) \in \mathbb{R}_{+} \times \mathbb{R}_{+}}$for $(t, s) \in \Lambda:=\left\{(t, s) \in \mathbb{R}_{+} \times \mathbb{R}_{+}: 0 \leq s \leq t<+\infty\right\}$.

This paper initiates the existence of solutions for evolution equations with an application of a generalization of the classical Darbo fixed point theorem, and the concept of measure of noncompactness in Fréchet spaces, The paper is organized as follows. In Section 2 some preliminary results are introduced. The main results is presented in Section 3, while the last section is devoted to an illustrative example.

Email addresses: said.abbas@univ-saida.dz, abbasmsaid@yahoo.fr (S. Abbas), amaria_ar@yahoo.fr (A. Arara), benchohra@univ-sba.dz (M. Benchohra), f_mesri@yahoo.fr (F. Mesri) 


\section{Preliminaries}

Let $I:=[0, T] ; T>0$. A measurable function $u: I \rightarrow E$ is Bochner integrable if and only if $\|u\|$ is Lebesgue integrable. For properties of the Bochner integral, see for instance, Yosida [19].

By $B(E)$ we denote the Banach space of all bounded linear operators from $E$ into $E$, with the norm

$$
\|N\|_{B(E)}=\sup _{\|u\|=1}\|N(u)\| .
$$

As usual, $L^{1}(I, E)$ denotes the Banach space of measurable functions $u: I \rightarrow E$ which are Bochner integrable and normed by

$$
\|u\|_{L^{1}}=\int_{0}^{T}\|u(t)\| d t
$$

As usual, by $\mathscr{C}:=C(I)$ we denote the Banach space of all continuous functions from $I$ into $E$ with the norm $\|\cdot\|_{\infty}$ defined by

$$
\|u\|_{\infty}=\sup _{t \in I}\|u(t)\| .
$$

In what follows, for the family $\{A(t), t \geq 0\}$ of closed densely defined linear unbounded operators on the Banach space $E$ we assume that it satisfies the following assumptions (see [3], p. 158).

$\left(P_{1}\right)$ The domain $D(A(t))$ is independent of $t$ and is dense in $E$,

$\left(P_{2}\right)$ For $t \geq 0$, the resolvent $R(\lambda, A(t))=(\lambda I-A(t))^{-1}$ exists for all $\lambda$ with $R e \lambda \leq 0$, and there is a constant $K$ independent of $\lambda$ and $t$ such that

$$
\|R(t, A(t))\| \leq K(1+|\lambda|)^{-1}, \text { for } \operatorname{Re} \lambda \leq 0,
$$

$\left(P_{3}\right)$ There exist constants $L>0$ and $0<\alpha \leq 1$ such that

$$
\left\|(A(t)-A(\theta)) A^{-1}(\tau)\right\| \leq L|t-\tau|^{\alpha}, \text { for } t, \theta, \tau \in I .
$$

Lemma 2.1. ([3], p. 159) Under assumptions $\left(P_{1}\right)-\left(P_{3}\right)$, the Cauchy problem

$$
u^{\prime}(t)-A(t) u(t)=0, t \in I, u(0)=y_{0},
$$

has a unique evolution system $U(t, s),(t, s) \in \Delta:=\{(t, s) \in J \times J: 0 \leq s \leq t \leq T\}$ satisfying the following properties:

1. $U(t, t)=I$ where $I$ is the identity operator in $E$,

2. $U(t, s) U(s, \tau)=U(t, \tau)$ for $0 \leq \tau \leq s \leq t \leq T$,

3. $U(t, s) \in B(E)$ the space of bounded linear operators on $E$, where for every $(t, s) \in \Delta$ and for each $u \in E$, the mapping $(t, s) \rightarrow U(t, s) u$ is continuous.

More details on evolution systems and their properties can be found in the books of Ahmed [3] and Pazy [17].

Let $C\left(\mathbb{R}_{+}\right)$be the Fréchet space of all continuous functions $v$ from $\mathbb{R}_{+}$into $E$, equipped with the family of seminorms

$$
\|v\|_{n}=\sup _{t \in[0, n]}\|v(t)\| ; n \in \mathbb{N},
$$

and the distance

$$
d(u, v)=\sum_{n=1}^{\infty} 2^{-n} \frac{\|u-v\|_{n}}{1+\|u-v\|_{n}} ; u, v \in C\left(\mathbb{R}_{+}\right)
$$

We recall the following definition of the notion of a sequence of measures of noncompactness [10, 11].

Definition 2.2. Let $\mathscr{M}_{X}$ be the family of all nonempty and bounded subsets of a Fréchet space $X$. A family of functions $\left\{\mu_{n}\right\}_{n \in \mathbb{N}}$ where $\mu_{n}: \mathscr{M}_{X} \rightarrow[0, \infty)$ is said to be a family of measures of noncompactness in the real Fréchet space $X$ if it satisfies the following conditions for all $B, B_{1}, B_{2} \in \mathscr{M}_{X}$ :

(a) $\left\{\mu_{n}\right\}_{n \in \mathbb{N}}$ is full, that is: $\mu_{n}(B)=0$ for $n \in \mathbb{N}$ if and only if $B$ is precompact,

(b) $\mu_{n}\left(B_{1}\right) \leq \mu_{n}\left(B_{2}\right)$ for $B_{1} \subset B_{2}$ and $n \in \mathbb{N}$,

(c) $\mu_{n}(\operatorname{Conv} B)=\mu_{n}(B)$ for $n \in \mathbb{N}$,

(d) If $\left\{B_{i}\right\}_{i=1, \cdots}$ is a sequence of closed sets from $\mathscr{M}_{X}$ such that $B_{i+1} \subset B_{i} ; i=1, \cdots$ and if $\lim _{i \rightarrow \infty} \mu_{n}\left(B_{i}\right)=0$, for each $n \in \mathbb{N}$, then the intersection set $B_{\infty}:=\cap_{i=1}^{\infty} B_{i}$ is nonempty.

\section{Some Properties:}

(e) We call the family of measures of noncompactness $\left\{\mu_{n}\right\}_{n \in \mathbb{N}}$ to be homogeneous if $\mu_{n}(\lambda B)=|\lambda| \mu_{n}(B)$; for $\lambda \in \mathbb{R}$ and $n \in \mathbb{N}$.

(f) If the family $\left\{\mu_{n}\right\}_{n \in \mathbb{N}}$ satisfied the condition $\mu_{n}\left(B_{1} \cup B_{2}\right) \leq \mu_{n}\left(B_{1}\right)+\mu_{n}\left(B_{2}\right)$, for $n \in \mathbb{N}$, it is called subadditive.

(g) It is sublinear if both conditions (e) and (f) hold.

(h) We say that the family of measures $\left\{\mu_{n}\right\}_{n \in \mathbb{N}}$ has the maximum property if

$$
\mu_{n}\left(B_{1} \cup B_{2}\right)=\max \left\{\mu_{n}\left(B_{1}\right), \mu_{n}\left(B_{2}\right)\right\}
$$


(i) The family of measures of noncompactness $\left\{\mu_{n}\right\}_{n \in \mathbb{N}}$ is said to be regular if and only if the conditions (a), (g) and (h) hold; (full sublinear and has maximum property).

Example 2.3. For $B \in \mathscr{M}_{X}, x \in B, n \in \mathbb{N}$ and $\varepsilon>0$, let us denote by $\omega^{n}(x, \varepsilon)$ for $n \in \mathbb{N}$; the modulus of continuity of the function $x$ on the interval $[0, n]$; that is,

$$
\omega^{n}(x, \varepsilon)=\sup \{|x(t)-x(s)|: t, s \in[0, n],|t-s| \leq \varepsilon\} .
$$

Further, let us put

$$
\begin{gathered}
\omega^{n}(B, \varepsilon)=\sup \left\{\omega^{n}(x, \varepsilon): x \in B\right\}, \\
\omega_{0}^{n}(B)=\lim _{\varepsilon \rightarrow 0^{+}} \omega^{n}(B, \varepsilon), \\
\bar{\alpha}^{n}(B)=\sup _{t \in[0, n]} \alpha(B(t)):=\sup _{t \in[0, n]} \alpha(\{x(t): x \in B\}),
\end{gathered}
$$

and

$$
\beta_{n}(B)=\omega_{0}^{n}(B)+\bar{\alpha}^{n}(B) .
$$

The family of mappings $\left\{\beta_{n}\right\}_{n \in \mathbb{N}}$ where $\beta_{n}: \mathscr{M}_{X} \rightarrow[0, \infty)$, satisfies the conditions $(a)-(d)$ from Definition 2.2 .

Definition 2.4. A nonempty subset $B \subset X$ is said to be bounded if

$$
\sup _{v \in X}\|v\|_{n}<\infty
$$

for $n \in \mathbb{N}$

Lemma 2.5. [9] If $Y$ is a bounded subset of Fréchet space $X$, then for each $\varepsilon>0$, there is a sequence $\left\{y_{k}\right\}_{k=1}^{\infty} \subset Y$ such that

$$
\mu_{n}(Y) \leq 2 \mu_{n}\left(\left\{y_{k}\right\}_{k=1}^{\infty}\right)+\varepsilon ; \text { for } n \in \mathbb{N} .
$$

Lemma 2.6. [15] If $\left\{u_{k}\right\}_{k=1}^{\infty} \subset L^{1}(I)$ is uniformly integrable, then $\mu_{n}\left(\left\{u_{k}\right\}_{k=1}^{\infty}\right)$ is measurable for $n \in \mathbb{N}$, and

$$
\mu_{n}\left(\left\{\int_{0}^{t} u_{k}(s) d s\right\}_{k=1}^{\infty}\right) \leq 2 \int_{0}^{t} \mu_{n}\left(\left\{u_{k}(s)\right\}_{k=1}^{\infty}\right) d s,
$$

for each $t \in[0, n]$.

Definition 2.7. Let $\Omega$ be a nonempty subset of a Fréchet space $X$, and let $A: \Omega \rightarrow X$ be a continuous operator which transforms bounded subsets of onto bounded ones. One says that A satisfies the Darbo condition with constants $\left(k_{n}\right)_{n \in \mathbb{N}}$ with respect to a family of measures of noncompactness $\left\{\mu_{n}\right\}_{n \in \mathbb{N}}$, if

$$
\mu_{n}(A(B)) \leq k_{n} \mu_{n}(B)
$$

for each bounded set $B \subset \Omega$ and $n \in \mathbb{N}$. If $k_{n}<1 ; n \in \mathbb{N}$ then $A$ is called a contraction with respect to $\left\{\mu_{n}\right\}_{n \in \mathbb{N}}$.

In the sequel we will make use of the following generalization of the classical Darbo fixed point theorem for Fréchet spaces.

Theorem 2.8. [10, 11] Let $\Omega$ be a nonempty, bounded, closed, and convex subset of a Fréchet space $F$ and let $V: \Omega \rightarrow \Omega$ be a continuous mapping. Suppose that $V$ is a contraction with respect to a family of measures of noncompactness $\left\{\mu_{n}\right\}_{n \in \mathbb{N}}$. Then $V$ has at least one fixed point in the set $\Omega$.

\section{Existence of mild solutions}

In this section, we present the main results for the global existence of solutions for our problem. Let us introduce the definition of the mild solution of the problem (1.1)-(1.2).

Definition 3.1. A continuous function $u(\cdot): I \rightarrow E$ is said a mild solution of the problem (1.1)-(1.2), if $u$ satisfies the following integral equation

$$
u(t)=U(t, 0) u_{0}+\int_{0}^{t} U(t, s) f(s, u(s)) d s, \quad \text { for each } t \in \mathbb{R}_{+} .
$$

Let us introduce the following hypotheses.

$\left(H_{1}\right)$ There exists a constant $M \geq 1$ such that

$$
\|U(t, s)\|_{B(E)} \leq M \text {; for every }(t, s) \in \Lambda .
$$

$\left(H_{2}\right)$ The function $t \mapsto f(t, u)$ is measurable on $\mathbb{R}_{+}$for each $u \in E$, and the function $u \mapsto f(t, u)$ is continuous on $E$ for a.e. $t \in \mathbb{R}_{+}$.

$\left(H_{3}\right)$ There exists a continuous function $p: \mathbb{R}_{+} \rightarrow[0, \infty)$ such that

$$
\|f(t, u)\| \leq p(t)(1+\|u\|) \text {; for a.e. } t \in \mathbb{R}_{+}, \text {and each } u \in E .
$$

$\left(H_{4}\right)$ For each bounded and measurable set $B \subset E$ and for each $t \in \mathbb{R}_{+}$, we have

$$
\mu(f(t, B)) \leq p(t) \mu(B)
$$

where $\mu$ is a measure of noncompactness on the Banach space $E$. 
For $n \in \mathbb{N}$, let

$$
p_{n}^{*}=\sup _{t \in[0, n]} p(t)
$$

and define on $C\left(\mathbb{R}_{+}\right)$the family of measure of noncompactness by

$$
\mu_{n}(D)=\sup _{t \in[0, n]} e^{-4 M p_{n}^{*} \tau t} \mu(D(t)),
$$

where $\tau>1$, and $D(t)=\{v(t) \in E: v \in D\} ; t \in[0, n]$.

Theorem 3.2. Assume that the hypotheses $\left(H_{1}\right)-\left(H_{4}\right)$ are satisfied, and $n M p_{n}^{*}<1$ for each $n \in \mathbb{N}$. Then the problem (1.1)-(1.2) has at least one mild solution.

Proof. Consider the operator $N: C\left(\mathbb{R}_{+}\right) \rightarrow C\left(\mathbb{R}_{+}\right)$defined by:

$$
(N u)(t)=U(t, 0) u_{0}+\int_{0}^{t} U(t, s) f(s, u(s)) d s
$$

Clearly, the fixed points of the operator $N$ are solution of the problem (1.1)-(1.2).

For any $n \in \mathbb{N}$, let $R_{n}$ be a positive real number with

$$
R_{n} \geq \frac{M\left\|u_{0}\right\|+n p_{n}^{*} M}{1-n M p_{n}^{*}}
$$

and we consider the ball

$$
B_{R_{n}}:=B\left(0, R_{n}\right)=\left\{w \in C\left(\mathbb{R}_{+}\right):\|w\|_{n} \leq R_{n}\right\} .
$$

For any $n \in \mathbb{N}$, and each $u \in B_{R_{n}}$ and $t \in[0, n]$ we have

$$
\begin{aligned}
\|(N u)(t)\| & \leq\|U(t, 0)\|_{B(E)}\left\|u_{0}\right\|+\int_{0}^{t}\|U(t, s)\|_{B(E)}\|f(s, u(s))\| d s \\
& \leq M\left\|u_{0}\right\|+M\left(\int_{0}^{t} p(s)(1+\|u(s)\|) d s\right) \\
& \leq M\left\|u_{0}\right\|+M\left(1+\|u\|_{n}\right) \int_{0}^{t} p(s) d s \\
& \leq M\left\|u_{0}\right\|+n M p_{n}^{*}\left(1+R_{n}\right) \\
& \leq R_{n} .
\end{aligned}
$$

Thus

$$
\|N(u)\|_{n} \leq R_{n}
$$

This proves that $N$ transforms the ball $B_{R_{n}}$ into itself. We shall show that the operator $N: B_{R_{n}} \rightarrow B_{R_{n}}$ satisfies all the assumptions of Theorem 2.8. The proof will be given in several steps.

Step 1. $N: B_{R_{n}} \rightarrow B_{R_{n}}$ is continuous.

Let $\left\{u_{k}\right\}_{k \in \mathbb{N}}$ be a sequence such that $u_{k} \rightarrow u$ in $B_{R_{n}}$. Then, for each $t \in[0, n]$, we have

$$
\begin{aligned}
\left\|\left(N u_{k}\right)(t)-(N u)(t)\right\| & \leq \int_{0}^{t}\|U(t, s)\|_{B(E)}\left\|f\left(s, u_{k}(s)\right)-f(s, u(s))\right\| d s \\
& \leq M \int_{0}^{t}\left\|f\left(s, u_{k}(s)\right)-f(s, u(s))\right\| d s
\end{aligned}
$$

Since $u_{k} \rightarrow u$ as $k \rightarrow \infty$, the Lebesgue dominated convergence theorem implies that

$$
\left\|N\left(u_{k}\right)-N(u)\right\|_{n} \rightarrow 0 \quad \text { as } k \rightarrow \infty .
$$

Step 2. $N\left(B_{R_{n}}\right)$ is bounded.

Since $N\left(B_{R_{n}}\right) \subset B_{R_{n}}$ and $B_{R_{n}}$ is bounded, then $N\left(B_{R_{n}}\right)$ is bounded. 
Step 3. For each bounded subset $D$ of $B_{R_{n}}, \mu_{n}(N(D)) \leq \ell_{n} \mu_{n}(D)$.

From Lemmas 2.5 and 2.6, for any $D \subset B_{R_{n}}$ and any $\varepsilon>0$, there exists a sequence $\left\{u_{k}\right\}_{k=0}^{\infty} \subset D$, such that for all $t \in[0, n]$, we have

$$
\begin{aligned}
\mu((N D)(t)) & =\mu\left(\left\{U(t, 0) u_{0}+\int_{0}^{t} U(t, s) f(s, u(s)) d s ; u \in D\right\}\right) \\
& \leq 2 \mu\left(\left\{\int_{0}^{t} U(t, s) f\left(s, u_{k}(s)\right) d s\right\}_{k=1}^{\infty}\right)+\varepsilon \\
& \leq 4 \int_{0}^{t} \mu\left(\|U(t, s)\|_{B(E)}\left\{f\left(s, u_{k}(s)\right)\right\}_{k=1}^{\infty}\right) d s+\varepsilon \\
& \leq 4 M \int_{0}^{t} \mu\left(\left\{f\left(s, u_{k}(s)\right\}_{k=1}^{\infty}\right) d s+\varepsilon\right. \\
& \leq 4 M \int_{0}^{t} p(s) \mu\left(\left\{u_{k}(s)\right\}_{k=1}^{\infty}\right) d s+\varepsilon \\
& \leq 4 M p_{n}^{*} \int_{0}^{t} e^{4 M p_{n}^{*} \tau s} e^{-4 M p_{n}^{*} \tau s} \mu\left(\left\{u_{k}(s)\right\}_{k=1}^{\infty}\right) d s+\varepsilon \\
& \leq \frac{\left(e^{4 M p_{n}^{*} \tau t}-1\right)}{\tau} \mu_{n}(D)+\varepsilon \\
& \leq \frac{e^{4 M p_{n}^{*} \tau t}}{\tau} \mu_{n}(D)+\varepsilon .
\end{aligned}
$$

Since $\varepsilon>0$ is arbitrary, then

$$
\mu((N D)(t)) \leq \frac{e^{4 M p_{n}^{*} \tau t}}{\tau} \mu_{n}(D)
$$

Thus

$$
\mu_{n}(N(D)) \leq \frac{1}{\tau} \mu_{n}(D)
$$

As a consequence of steps 1 to 3 together with Theorem 2.8, we can conclude that $N$ has at least one fixed point in $B_{R_{n}}$ which is a mild solution of problem (1.1)-(1.2).

\section{An example}

As an application of our results, we consider the following functional evolution problem of the form

$$
\left\{\begin{array}{cc}
\frac{\partial z}{\partial t}(t, x)=a(t, x) \frac{\partial^{2} z}{\partial x^{2}}(t, x)+Q(t, z(t, x)) ; & t \in \mathbb{R}_{+}, x \in[0, \pi], \\
z(t, 0)=z(t, \pi)=0 ; & t \in \mathbb{R}_{+} \\
z(0, x)=\Phi(x) ; & x \in[0, \pi],
\end{array}\right.
$$

where $a(t, x): \mathbb{R}_{+} \times[0, \pi] \rightarrow \mathbb{R}$ is a continuous function and is uniformly Hölder continuous in $t, Q: \mathbb{R}_{+} \times \mathbb{R} \rightarrow \mathbb{R}$ and $\Phi:[0, \pi] \rightarrow \mathbb{R}$ are continuous functions.

Consider $E=L^{2}([0, \pi], \mathbb{R})$ and define $A(t)$ by $A(t) w=a(t, x) w^{\prime \prime}$ with domain

$$
D(A)=\left\{w \in E: w, w^{\prime} \text { are absolutely continuous, } w^{\prime \prime} \in E, w(0)=w(\pi)=0\right\} .
$$

Then $A(t)$ generates an evolution system $U(t, s)$ (see [12]).

For $x \in[0, \pi]$, we have

$$
\begin{gathered}
y(t)(x)=z(t, x) ; \quad t \in \mathbb{R}_{+}, \\
f(t, y)(x)=Q(t, z(t, x)) ; \quad t \in \mathbb{R}_{+},
\end{gathered}
$$

and

$$
u_{0}(x)=\Phi(x) ; \quad x \in[0, \pi]
$$

Thus, under the above definitions of $f, u_{0}$ and $A(\cdot)$, the system (4.1) can be represented by the functional evolution problem (1.1)-(1.2). Furthermore, more appropriate conditions on $Q$ ensure the hypotheses $\left(H_{1}\right)-\left(H_{4}\right)$. Consequently, Theorem 3.2 implies that the evolution problem (4.1) has at least one mild solution. 


\section{References}

[1] S. Abbas, W. Albarakati and M. Benchohra, Successive approximations for functional evolution equations and inclusions, J. Nonlinear Funct. Anal., Vol. 2017 (2017), Article ID 39, pp. 1-13.

[2] S. Abbas and M. Benchohra, Advanced Functional Evolution Equations and Inclusions, Springer, Cham, 2015.

[3] N. U. Ahmed, Semigroup Theory with Applications to Systems and Control, Pitman Research Notes in Mathematics Series, 246. Longman Scientific \& Technical, Harlow; John Wiley \& Sons, New York, 1991.

[4] S. Baghli and M. Benchohra, Global uniqueness results for partial functional and neutral functional evolution equations with infinite delay, Differential Integral Equations, 23 (2010), 31-50.

[5] S. Baghli and M. Benchohra, Multivalued evolution equations with infinite delay in Fréchet spaces, Electron. J. Qual. Theo. Differ. Equ. 2008, No. 33, 24 pp.

[6] A. Baliki and M. Benchohra, Global existence and asymptotic behaviour for functional evolution equations, J. Appl. Anal. Comput. 4 (2) (2014), $129-138$.

[7] A. Baliki and M. Benchohra, Global existence and stability for neutral functional evolution equations, Rev. Roumaine Math. Pures Appl. LX (1) (2015), $71-82$

[8] M. Benchohra and I. Medjadj, Global existence results for second order neutral functional differential equation with state-dependent delay. Comment. Math. Univ. Carolin. 57 (2016), 169-183.

[9] D. Bothe, Multivalued perturbation of m-accretive differential inclusions, Isr. J. Math. 108 (1998), 109-138.

[10] S. Dudek, Fixed point theorems in Fréchet Algebras and Fréchet spaces and applications to nonlinear integral equations, Appl. Anal. Discrete Math., 11 (2017), 340-357.

[11] S. Dudek and L. Olszowy, Continuous dependence of the solutions of nonlinear integral quadratic Volterra equation on the parameter, J. Funct. Spaces, V. 2015, Article ID 471235, 9 pages.

[12] A. Freidman, Partial Differential Equations, Holt, Rinehat and Winston, New York, 1969.

[13] M. Frigon and A. Granas, Résultats de type Leray-Schauder pour des contractions sur des espaces de Fréchet, Ann. Sci. Math. Québec 22 (2) (1998), $161-168$.

[14] S. Heikkila and V. Lakshmikantham, Monotone Iterative Technique for Nonlinear Discontinuous Differential Equations, Marcel Dekker Inc., New York, 1994.

[15] H. Mönch, Boundary value problems for nonlinear ordinary differential equations of second order in Banach spaces, Nonlinear Anal. 4(1980), 985-999.

[16] L. Olszowy and S. Wedrychowicz, Mild solutions of semilinear evolution equation on an unbounded interval and their applications, Nonlinear Anal. 72 (2010), 2119-2126.

[17] A. Pazy, Semigroups of Linear Operators and Applications to Partial Differential Equations, Springer-Verlag, New York, 1983

[18] J. Wu, Theory and Applications of Partial Functional Differential Equations, Applied Mathematical Sciences 119, Springer-Verlag, New York, 1996.

[19] K. Yosida, Functional Analysis, ${ }^{\text {th }}$ edn. Springer-Verlag, Berlin, 1980. 